\title{
Evolutionarily stable strategies for defensive switching
}

\begin{abstract}
This paper concentrates on the study of ecological stability for guaranteeing evolutionarily stable strategies in a two-predator and one-prey model system. It is assumed that the prey has defensive switching property of predation-avoidance which can be characterized by two parameters: the relative alertness of the prey against a predator, $\mathrm{u}$, and the intensity of defensive switching, $\mathrm{n}$. Assuming that the system is encountered with another immigrant prey with altered defensive switching parameters one after another, the conditions of replacement of the former occupant prey by an immigrant are studied. It is shown that the system eventually attains the evolutionarily stable state such that no entry of an immigrant prey is possible. Unless some trade-off relation exists between $u$ and $n$, the evolutionarily stable state is not unique, but it is given by a set of parameters $\{\mathrm{u}, \mathrm{n}\}$ which form a curve designated "ESS line". Thus, it is seen that if a number of two-predator and one-prey systems are allowed to evolve independently, each system will reach a different state on the "ESS line". Finally, we discuss a strategy that leads to optimal (minimum) prey consumption by both predators in relation to the "ESS line".
\end{abstract}

Keyword: Predator-prey interactions, Defensive switching, Evolutionarily stable strategies, Coexistence of species 